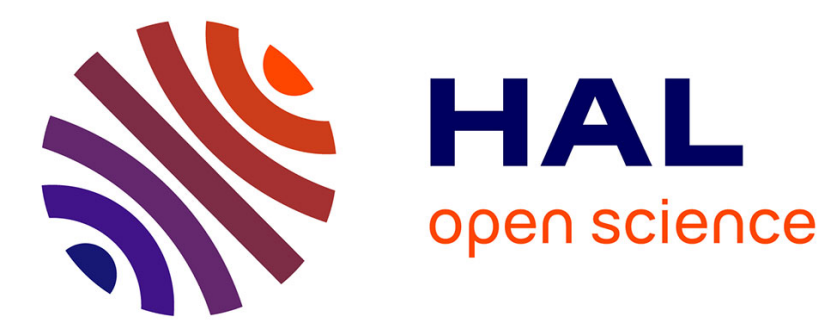

\title{
Imidazolium Ionic Liquid Interfaces with Vapor and Graphite: Interfacial Tension and Capacitance from Coarse-Grained Molecular Simulations
}

Céline Merlet, Mathieu Salanne, Benjamin Rotenberg, Paul A Madden

\section{To cite this version:}

Céline Merlet, Mathieu Salanne, Benjamin Rotenberg, Paul A Madden. Imidazolium Ionic Liquid Interfaces with Vapor and Graphite: Interfacial Tension and Capacitance from CoarseGrained Molecular Simulations. Journal of Physical Chemistry C, 2011, 115 (33), pp.16613-16618. 10.1021/jp205461g . hal-00854030

\section{HAL Id: hal-00854030 \\ https://hal.sorbonne-universite.fr/hal-00854030}

Submitted on 16 Nov 2018

HAL is a multi-disciplinary open access archive for the deposit and dissemination of scientific research documents, whether they are published or not. The documents may come from teaching and research institutions in France or abroad, or from public or private research centers.
L'archive ouverte pluridisciplinaire HAL, est destinée au dépôt et à la diffusion de documents scientifiques de niveau recherche, publiés ou non, émanant des établissements d'enseignement et de recherche français ou étrangers, des laboratoires publics ou privés. 


\title{
Imidazolium Ionic Liquid Interfaces with Vapor and Graphite: Interfacial Tension and Capacitance from Coarse-Grained Molecular Simulations
}

\author{
Céline Merlet ${ }^{1}$, Mathieu Salanne ${ }^{1}$, Benjamin Rotenberg ${ }^{1}$, and Paul Madden ${ }^{2}$ \\ 1 UPMC Univ Paris 06 and CNRS, UMR 7195, \\ PECSA, F-75005, Paris, France and \\ 2 Department of Materials, University of Oxford, \\ Parks Road, Oxford OX1 3PH, UK
}

\begin{abstract}
A recently developed coarse-grained model (J. Phys. Chem. B, 2010, 114, 12629-12631), previously validated against experimental data for a number of bulk properties, is used in molecular dynamics simulations of two different interfaces involving the ionic liquid $[\mathrm{BMI}]\left[\mathrm{PF}_{6}\right]$. First, simulations of the liquid-vapor interface demonstrate that the model is able to predict the surface tension of the fluid (for which we obtain a value of $39.4 \mathrm{mN} \cdot \mathrm{m}^{-1}$ at $400 \mathrm{~K}$ ). Second, simulations were performed at constant potential differences applied between two graphite electrodes. From simulations with different applied potentials, the differential capacitances of the positive and negative electrodes can be calculated. It appears that both capacitances $\left(\mathrm{C}_{+}=3.9 \mu \mathrm{F} . \mathrm{cm}^{-2}\right.$ for the positive electrode and $\mathrm{C}_{-}=4.8 \mu \mathrm{F} . \mathrm{cm}^{-2}$ for the negative electrode) agree very well with simulations results obtained with an all-atom model. The coarse-grained model also reproduces accurately the two-dimensional structure observed at the graphite-ionic liquid interface, namely a defective hexagonal lattice with a lattice spacing of approximately $10 \AA$.
\end{abstract}




\section{INTRODUCTION}

Ionic liquids are currently under intense examination as potential electrolytes for future capacitors [1, 2, 3] as they present a number of highly appropriate properties such as low vapor pressure, low combustibility, high thermal stability, good ionic conductivity and wide electrochemical windows. Moreover, their properties are in principle tunable thanks to the wide variety of cations and anions that can be mixed. Nevertheless, synthesizing, purifying and characterizing new ionic liquids remains expensive and systematic procedures to evaluate new materials are out of reach. Furthermore, a number of experimental observations are not understood, mainly because they are the result of microscopic phenomena which cannot be probed experimentally. Thus, simulation appears as an essential tool to explain what is observed in experiments and to design new materials with improved performances.

One particular experimental result that still suffers from a lack of explanation is the anomalously high value of the capacitance at nanoporous carbon electrodes [2, 4, 5, 6, 7]. Understanding this increase could lead to the design of new materials for future capacitors with improved performances. Furthermore, there is an issue about the shape of the capacitance curve when the external potential is varied. Some experimental [8, 9] and theoretical [10, 11] results suggest camel-shaped and bell-shaped variations for the capacitance but others tend to show that the capacitance is constant [12]. The capacitance curve can depend on several processes with different time constants as seen at the interface between $\mathrm{Au}(111)$ and 1-ethyl3-methylimidazolium tris(pentafluoroethyl)-trifluorophosphate [13]. Thus, a representation of the processes happening at the atomic scale in these systems is needed.

Nowadays, ionic liquids are commonly studied by molecular dynamics and Monte Carlo simulations, and most of the structural, thermodynamic and dynamical bulk properties of these systems are well reproduced by recent force fields [14, 15, 16]. Interfaces involving ionic liquids are also well represented in the literature and molecular dynamics have already proven efficient in giving insights on liquid-solid interfaces and the capacitive behaviors of systems with simple electrodes [17, 18, 19]. Most of the liquid-electrode interfaces are studied with the electrode potential generated by holding the electrode at a constant charge [20] which is assigned equal values on equivalent sites. This may well be a reasonable approximation for planar electrodes but will become problematic for more complex geometries for which it is necessary to obtain charges which are consistent with the applied potential and 
the shape and dielectric properties of the electrode and the charge distribution of the ions across the interface [21].

To explain the capacitance behavior for nanoporous carbons, it is necessary to find a model sufficiently accurate to reproduce structural, dynamical and capacitive properties but simple enough to allow for calculations on complex systems with nanoporous electrodes. A recently developed four-site model for the ionic liquid $[\mathrm{BMI}]\left[\mathrm{PF}_{6}\right][22$ is examined in this work as a candidate for use in molecular dynamics simulations in complex systems. To deal with the problem of developing a consistent description of the charge on the roughly-shaped electrodes, we examine the use of a method which allows the electrodes to be treated as polarizable and maintained at a constant electrical potential, with the charges being determined self-consistently [23].

The remainder of this article is organized as follows. In section 2 we introduce the model chosen to describe the ionic liquid and the carbon electrodes. In section 3 we show that the model is able to predict liquid-vapor interfacial properties accurately. In sections 4 and 5 we demonstrate that the model is also efficient for the prediction of capacitive and structural properties of the interface between planar graphite electrodes and the ionic liquid.

\section{MODEL AND SIMULATION METHODS}

The ionic liquid is represented by a coarse-grained model developed by Roy and Maroncelli which accurately predicts a number of bulk properties [22] such as molar volume, isothermal compressibility, viscosity and diffusion. This model is actually an improvement of a previous one published by the same authors which showed poorer dynamical properties [24. The most important difference between the two models is the use of reduced charges, i.e. all the charges of the first coarse-grained model were scaled by a same factor. This approach was also used successfully in other systems [14, 25, 26, 27] and it is thought that reducing charges compensates for the missing polarization term in an average way in these force fields. Going beyond the topic of ionic liquids, the Molecular Dynamics in Electronic Continuum model provides a theoretical framework for systematic accounting of the effects of electronic polarization through the use of rescaled charges [28]. A recent publication describes a methodology to calculate the scaling factor from DFT calculations of the electrostatic potential in liquid phase configurations [29]. Van der Waals attraction and repulsion are 


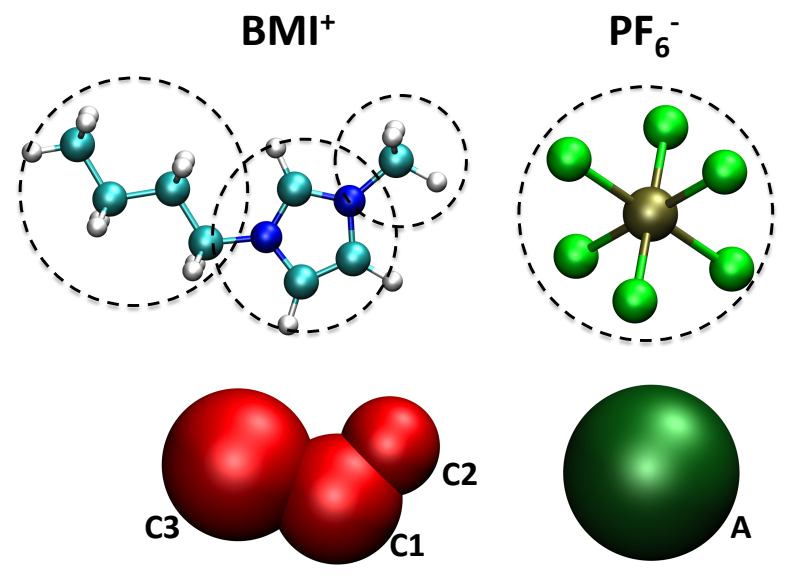

Figure 1: Schematic representation of the coarse-grained model of $[\mathrm{BMI}]\left[\mathrm{PF}_{6}\right]$.

\begin{tabular}{|c|c|c|c|c|c|c|c|}
\hline Site & $\begin{array}{c}\mathrm{x} \\
(\AA)\end{array}$ & $\begin{array}{c}\mathrm{y} \\
(\AA)\end{array}$ & $\begin{array}{c}\mathrm{z} \\
(\AA)\end{array}$ & $\begin{array}{c}\mathrm{M} \\
\left(\mathrm{g} . \mathrm{mol}^{-1}\right)\end{array}$ & $\begin{array}{c}\sigma_{i} \\
(\AA)\end{array}$ & $\begin{array}{c}\varepsilon_{i} \\
\left(\mathrm{~kJ} . \mathrm{mol}^{-1}\right)\end{array}$ & $\begin{array}{c}q_{i} \\
(\mathrm{e})\end{array}$ \\
\hline $\mathrm{C} 1$ & 0 & -0.527 & 1.365 & 67.07 & 4.38 & 2.56 & 0.4374 \\
\hline $\mathrm{C} 2$ & 0 & 1.641 & 2.987 & 15.04 & 3.41 & 0.36 & 0.1578 \\
\hline $\mathrm{C} 3$ & 0 & 0.187 & -2.389 & 57.12 & 5.04 & 1.83 & 0.1848 \\
\hline $\mathrm{A}$ & 0 & 0 & 0 & 144.96 & 5.06 & 4.71 & -0.7800 \\
\hline
\end{tabular}

Table I: Force-field parameters of the coarse-grained model of $[\mathrm{BMI}]\left[\mathrm{PF}_{6}\right]$ [22]. Site-site interaction energies are given by the sum of a Lennard-Jones potential and coulombic interactions $u_{i j}\left(r_{i j}\right)=$ $4 \varepsilon_{i j}\left[\left(\frac{\sigma_{i j}}{r_{i j}}\right)^{12}-\left(\frac{\sigma_{i j}}{r_{i j}}\right)^{6}\right]+\frac{q_{i} q_{j}}{4 \pi \varepsilon_{0} r_{i j}}$ where $r_{i j}$ is the distance between sites, $\varepsilon_{0}$ is the permittivity of free space and crossed parameters are calculated by Lorentz-Berthelot mixing rules. The parameters for the carbon atoms of the graphite electrodes are $\sigma_{\mathrm{C}}=3.37 \AA$ and $\varepsilon_{\mathrm{C}}=0.23 \mathrm{~kJ} . \mathrm{mol}^{-1}[30$.

described using pairwise additive Lennard-Jones interactions. The parameters of the coarsegrained model developed by Roy and Maroncelli [22] to represent [BMI] $\left[\mathrm{PF}_{6}\right]$ are recalled in table 1 and a schematic representation of the ionic liquid is given in figure 1 . For the graphite electrodes, potential parameters for carbon atoms were set equal to $\sigma_{\mathrm{C}}=3.37 \AA$ and $\varepsilon_{\mathrm{C}}=$ $0.23 \mathrm{~kJ} . \mathrm{mol}^{-1}$ following Cole and Klein [30]. All the cross parameters were obtained using the Lorentz-Berthelot mixing rules.

The electrodes are modelled following a method developed by Reed et al. [23], following a proposal by Siepmann and Sprik [31], which consists in determining the charge on each 
electrode atom at each MD step by requiring that the potential on this atom is constant and equal to a specified value. This condition therefore means that the electrode polarizes in the same way as a metal, where the potential inside the metal is a constant. The results obtained with this construction will be contrasted with results obtained by assigning constant charges to the electrode atoms. In the constant potential method, the electrode atom charges, represented by gaussian distributions centered on the carbon atoms, are obtained by minimizing the expression:

$$
U=\sum_{i} q_{i}(t)\left[\frac{\Psi_{i}\left(\left\{q_{j}(t)\right\}\right)}{2}+\frac{q_{i}(t) \kappa}{\sqrt{2 \pi}}-\Psi^{0}\right],
$$

where $q_{i}$ is the charge on electrode atom $i, \kappa$ is the width of the gaussian distributions ( $\kappa$ $=0.5055 \AA$ in this work $), \Psi_{i}\left(\left\{q_{j}\right\}\right)$ is the potential at position $i$ due to all the ions in the electrolyte and the other electrode atoms, the second term originates from the interaction of the gaussian distribution with itself and $\Psi^{0}$ is the externally applied potential. Instead of minimizing this expression using a conjugate gradient method only, we use a two-step procedure where the first step is a prediction of the charges by a polynomial expansion, using the previous steps [32, 33], and the second step is a conjugate gradient minimization. This allows a speed-up by a factor of 2 compared to conjugate-gradient minimization only. This representation of the electrodes has been successfully applied to the interface between the molten salt $\mathrm{LiCl}$ and aluminium electrodes [34, 35].

All the simulations were conducted with a time step of 2 fs and by applying a Nosé-Hoover thermostat with a weak relaxation time constant of 10 ps to maintain a temperature of $400 \mathrm{~K}$. All the simulation cells are orthorhombic with cell lengths such that $L_{x} \sim L_{y}<L_{z}$ The electrostatic interactions are treated according to the periodic boundary conditions adopted (3D for liquid-vapor simulations, 2D for liquid-graphite simulations). The 2D Ewald summation is done as described in reference [23] and following recommendations of reference [36].

\section{LIQUID-VAPOR INTERFACE: SURFACE TENSION}

The coarse-grained model developed by Roy and Maroncelli has proven to be reliable for a number of bulk properties but has not yet been tested for interfacial properties. The simplest interface is the one between the ionic liquid and vacuum. The surface tension is a good test of the accuracy of the model for liquid-vapor interfaces as this property has 
been well studied by experiments [37, 38] and simulations [14, 39]. From simulations of an interface between $[\mathrm{BMI}]\left[\mathrm{PF}_{6}\right]$ and vacuum, it is possible to calculate the surface tension of the ionic liquid using the pressure tensor components [40, 41]:

$$
\gamma=\frac{L z}{2}\left(<\Pi_{z z}>-\frac{<\Pi_{x x}+\Pi_{y y}>}{2}\right) .
$$

The system consists in a slab (parallel to the xy plane) of ionic liquid sandwiched between two vacuum regions simulated with 3D periodic boundary conditions for $1 \mathrm{~ns}$. The simulations were conducted with an orthorhombic cell with lengths such that $L_{x} \sim L_{y} \neq$ $L_{z}$. The system contains 320 ion pairs for the first simulations when varying $L_{z}$ and 1280 ion pairs for the last simulation when $L_{x}$ and $L_{y}$ are changed.

In a first step, we analyze the effect of 3D periodic boundary conditions on the surface tension. In particular, there is a need to verify that the periodic slabs of ionic liquid do not interact with each other. In practice, when calculating the surface tension with different lengths in the $\mathrm{z}$ direction, it appears that there is a minimum length from which interactions can be considered negligible. Simulations were done with three different lengths $L_{z}$, i.e. increasing the vacuum region size and keeping $L_{x}$ and $L_{y}$ constant. The resulting surface tensions are $14.6 \mathrm{mN} . \mathrm{m}^{-1}, 26.0 \mathrm{mN} . \mathrm{m}^{-1}$ and $26.0 \mathrm{mN} . \mathrm{m}^{-1}$ for $L_{z}$ equal to $300 \AA, 400 \AA$ and $500 \AA$ respectively. It can thus be concluded that $500 \AA$ is a sufficient length to avoid interactions between the slabs in the $\mathrm{z}$ direction.

In a second step, we analyze the finite-size effect in our system. Calculations of interfacial properties of fluids are subject to potential errors due to finite-size effects. The capillary wave theory suggests that the surface tension converges to the macroscopic value following [42]:

$$
\gamma\left(L_{x}\right)=\gamma_{\infty}+\frac{\mathrm{C}}{L_{x}^{2}},
$$

where $\mathrm{C}$ is a constant and $\gamma_{\infty}$ is the macroscopic observable (when $L_{x}=L_{y}$ ). This prediction, first established for van der Waals fluids, is also applicable to simple molten salts as demonstrated for KI [43]. Recent studies show that for some liquids, the surface tension behaves somewhat differently [44]:

$$
\gamma\left(L_{x}\right)=\mathrm{A}_{0}+\mathrm{A}_{1} \frac{\exp \left(-\alpha_{c} L_{x}\right)}{L_{x}} \cos \left(\mathrm{A}_{2} L_{x}+\mathrm{A}_{3}\right),
$$

where $\alpha_{c}$ is a decay length and $\mathrm{A}_{\mathrm{i}}$ are constants. In both cases, when the interface area is larger than a threshold value, finite-size effects can be neglected. According to GonzálezMelchor et al. [44, the minimum length one has to consider is approximately equal to 
$10 \sigma_{ \pm}$with $\sigma_{ \pm}$being the diameter of cations and anions. For the model used in this work, the diameter of the ions is around $5 \AA$ which means that the lengths, $L_{x}=32.2 \AA$ and $L_{y}=34.4 \AA$ which were used in the previous simulations are too small to avoid finitesize dependency. The surface tension was thus evaluated for a system with a four times larger interface area $(72.0 \AA \times 77.0 \AA)$ and with a $500 \AA$ length in the $\mathrm{z}$ direction. We then obtained a value of $39.4 \mathrm{mN} . \mathrm{m}^{-1}$ which is in very good agreement with experimental data $\left(40.8 \mathrm{mN} . \mathrm{m}^{-1}\right.$ at $393 \mathrm{~K}$ [37], $41.07 \mathrm{mN} . \mathrm{m}^{-1}$ at $343.15 \mathrm{~K}$ [38]). The coarse-grained model performs even better than previously described all-atom models which overestimate (47 mN.m ${ }^{-1}$ [14], $74 \mathrm{mN} \cdot \mathrm{m}^{-1}$ [45]), or underestimate $\left(37.3 \mathrm{mN} \cdot \mathrm{m}^{-1}\right.$ [39]) the surface tension of $[\mathrm{BMI}]\left[\mathrm{PF}_{6}\right]$ at room temperature.

\section{DIFFERENTIAL CAPACITANCE}

The $[\mathrm{BMI}]\left[\mathrm{PF}_{6}\right]$-graphite interface has now been well characterized in various studies. It therefore provides us a good test of the ability of the coarse-grained model to predict capacitive and interfacial properties before tackling more complex interfaces, such as the ones involving nanoporous electrodes. Simulations were conducted with $\mathrm{BMI}^{+}$and $\mathrm{PF}_{6}^{-}$ ions confined between planar graphite electrodes using the following conditions. A typical simulation cell contains a slice of liquid $[\mathrm{BMI}]\left[\mathrm{PF}_{6}\right]$ (320 ion pairs) between two graphite electrodes (see figure 2). The graphite electrodes are made of three planes of 416 atoms each with a constant distance between atoms equal to $\mathrm{d}_{\mathrm{CC}}=1.43 \AA$. The simulation cell is orthorhombic with dimensions $32.2 \AA \times 34.4 \AA \times 123.2 \AA$.

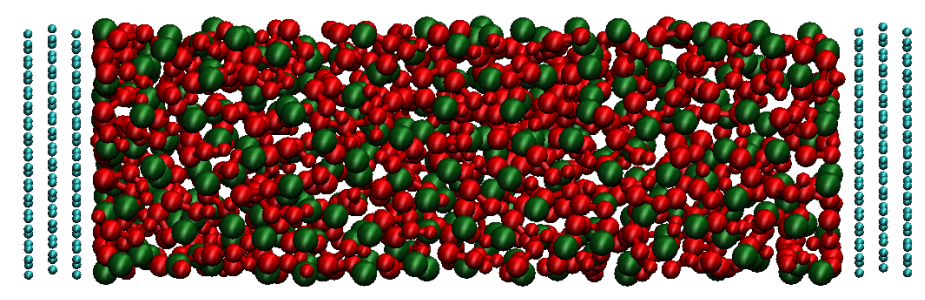

Figure 2: Snapshot of the simulation system: ionic liquid confined between graphite walls.

We perform simulations with nine potential differences ranging from $0.0 \mathrm{~V}$ to $4.0 \mathrm{~V}$. When a new potential difference is set between the electrodes, the system is allowed to equilibrate for 200 ps before collecting data during 1 ns. For all the simulations, the potential difference 
is applied so that the potential applied to the positive electrode $\Psi^{+}$is equal to the opposite of the negative electrode $\Psi^{-}$, i.e.:

$$
\Psi^{+}=-\Psi^{-}=\frac{\Delta \Psi^{0}}{2}
$$

The highest considered potential difference was chosen to match with the experimental electrochemical stability window of $[\mathrm{BMI}]\left[\mathrm{PF}_{6}\right][1]$.

The differential capacitance of the two liquid-graphite interfaces can then be determined from the mean surface charges on the electrodes and the electrostatic potential profile across the cell which is given by Poisson's equation:

$$
\begin{aligned}
\Psi(z) & =\Psi_{q}(z) \\
& =\Psi_{q}\left(z_{0}\right)-\frac{1}{\varepsilon_{0}} \int_{z_{0}}^{z} d z^{\prime} \int_{-\infty}^{z^{\prime}} d z^{\prime \prime} \rho_{q}\left(z^{\prime \prime}\right)
\end{aligned}
$$

where $z_{0}$ is a reference point inside the left-hand electrode with $\Psi_{q}\left(z_{0}\right)=\Psi^{+}$and $\rho_{q}(z)$ is the charge density across the cell, which includes the contributions from the electrode atoms as well as from the charges on the sites of the ionic liquid molecules. The profiles obtained (see figure 3) are consistent with the ones obtained from other simulations of this type with molten salts [34]. The potential of the bulk $\Psi_{b u l k}$, is given by the plateau in the bulk liquid region, and the potential drop across each interface $\Delta \Psi^{ \pm}=\Psi^{ \pm}-\Psi_{\text {bulk }}$ can be extracted from such profiles and used to characterize the interfaces.

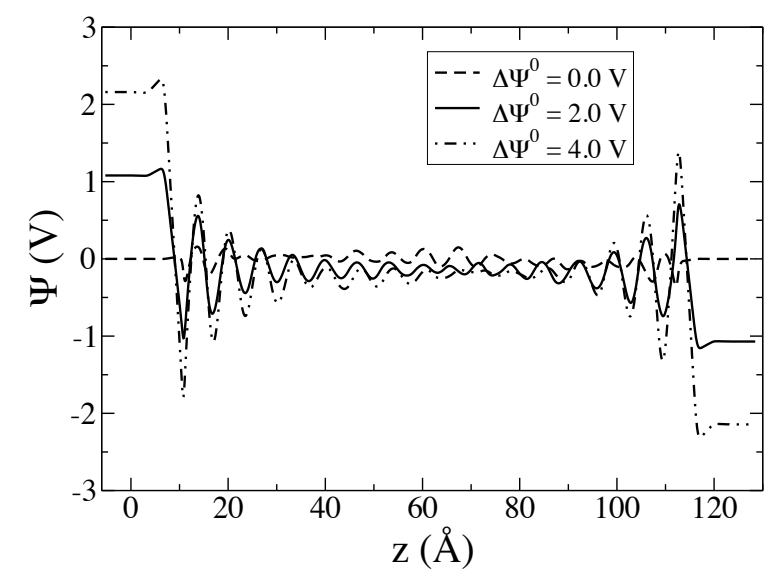

Figure 3: Poisson potential profiles across the simulation for three of the nine potential differences applied. The potential in the bulk region is constant and noted $\Psi_{\text {bulk }}$. 
The differential capacitances of the positive and negative electrodes are obtained by differentiation of the surface charge with respect to the potential drop across the interface (see figure 4):

$$
\mathrm{C}_{\text {diff }}=\frac{\partial \sigma}{\partial \Delta \Psi}
$$

It can be noted that the variation of the surface charge as a function of the potential

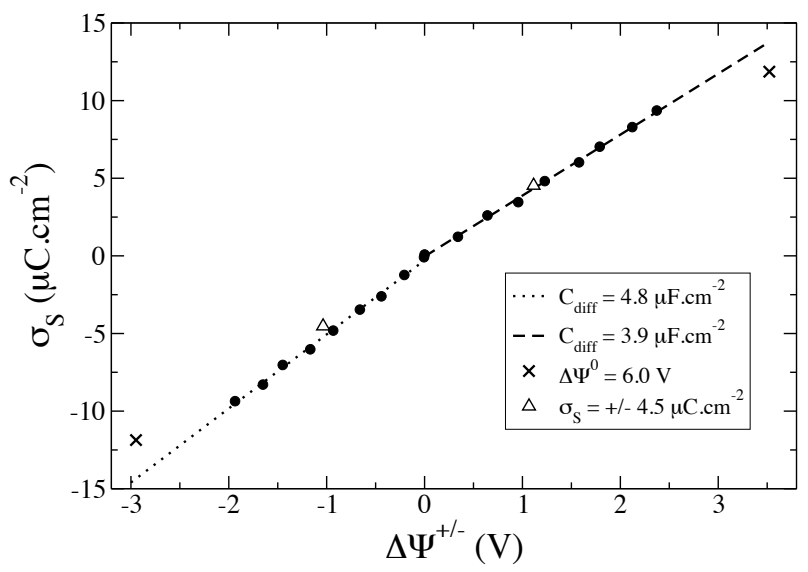

Figure 4: Surface charge as a function of the potential drop across the interface $\Delta \Psi=\Psi^{ \pm}-$ $\Psi^{b u l k}$. Two linear régimes are observed and differential capacitances for the positive and negative electrode are given by the corresponding slopes. Linear regressions are extrapolated to show that the simulated points for $\Delta \Psi^{0}=6.0 \mathrm{~V}$ do not lie on these lines. Points obtained with a constant charge of $\pm 4.5 \mu \mathrm{C} . \mathrm{cm}^{-2}$ compare well with constant potential results.

drop across the interface shows two régimes which are clearly linear, indicating that the differential capacitance is constant there, as currently observed in experiments with supercapacitors [4, 6]. Another quantity that can be determined from this plot is the potential of zero charge (PZC) which is zero for this system, in agreement with previous simulations on $[\mathrm{BMI}]\left[\mathrm{PF}_{6}\right][19$, 46]. Non-trivial values of the PZC were obtained in molten salt simulations of this type where the cations and anions differed in size [35]. This value, which is an absolute potential, cannot be compared directly with experimentally measured PZC as the experimental quantity is always given with respect to a reference electrode.

The differential capacitances obtained to the positive $\left(\mathrm{C}_{+}\right)$and negative $\left(\mathrm{C}_{-}\right)$sides of the PZC are summarized in table II. The difference between these values is linked to the different shapes of the ions and can also be noted in a plot of the ionic densities at the 
positive and negative electrodes (see figure 5). The differential capacitances calculated in

\begin{tabular}{|c|c|c|}
\hline Source & $\mathrm{C}_{+}\left(\mu \mathrm{F} . \mathrm{cm}^{-2}\right)$ & $\mathrm{C}_{-}\left(\mu \mathrm{F} . \mathrm{cm}^{-2}\right)$ \\
\hline This work & 3.9 & 4.8 \\
\hline Kislenko et al. [17] & 3.7 & 4.6 \\
\hline Feng et al. [19] & 4.40 & 4.09 \\
\hline
\end{tabular}

Table II: Comparison of differential capacitances obtained by different simulations. In this work, the molecular dynamics are conducted with a coarse-grained model at constant potential difference with polarizable electrodes whereas in the other publications cited, the simulations use an all-atom model and constant charge electrodes.

this work are in excellent agreement with the ones obtained by Kislenko et al. [17] with an all-atom model and a constant charge of $\pm 8.2 \mu \mathrm{C} . \mathrm{cm}^{-2}$ but differ from the results of

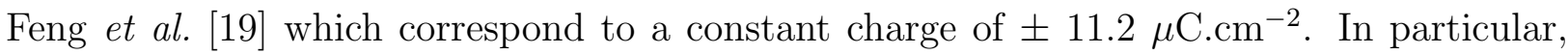
$\mathrm{C}_{-}$is greater than $\mathrm{C}_{+}$for this work but the results of reference [19] show the contrary. We underline here the fact that, for the present system, the highest potential difference, $\Delta \Psi^{0}$ $=4.0 \mathrm{~V}$, induces a mean surface charge of $\pm 9.4 \mu \mathrm{C} . \mathrm{cm}^{-2}$. Because this potential difference corresponds to the electrochemical stability window of [BMI][PF 6$]$ [1], it appears that a surface charge of $\pm 8.2 \mu \mathrm{C} . \mathrm{cm}^{-2}$ is realistic, while $\pm 11.2 \mu \mathrm{C} . \mathrm{cm}^{-2}$ might be too large to evaluate the differential capacitance of the system. To test this assumption, we performed a simulation in which a potential difference of $6.0 \mathrm{~V}$ was applied between the electrodes. This potential difference induces a surface charge of $11.9 \mu \mathrm{C} . \mathrm{cm}^{-2}$ on the graphite electrodes. Points for this simulation are also reported in figure 4 demonstrating that they are outside the linear régimes. The high surface charge applied in the simulations of Feng et al. is thus the explanation of the contradictory results.

The good agreement between our results obtained with polarizable electrodes and previous results derived from constant charge simulations suggest that, for this system, constant charge simulations are sufficiently accurate to reproduce the capacitive behavior of the ionic liquid-planar graphite interface. To directly test, we performed a simulation with a constant charge of $\pm 4.5 \mu \mathrm{C} \cdot \mathrm{cm}^{-2}$ on each electrode, the charge being distributed uniformly on all the atoms of the graphite plane closest to the interface. From the very similar value of the differential capacitance obtained in these calculations (figure 4), we confirm that, for planar 
electrodes, the constant charge simulations compare well with constant potential simulations (see figure 4). We recall here that the present work is directed at validating techniques for a coarse-grained model for $[\mathrm{BMI}]\left[\mathrm{PF}_{6}\right]$ for future use in simulations with porous electrodes, in the latter the constant potential treatment, which allows the electrode charges to be determined self-consistently, is crucial [21. We can thus conclude, in view of the present results, that the constant potential method has not introduced any new aspects to the results obtained at a planar electrode, despite the fact that it implicitly treats the graphite electrode as metallic.

\section{STRUCTURAL PROPERTIES AT THE LIQUID-GRAPHITE INTERFACE}

We showed in the previous section that the four-site model of Roy and Maroncelli [22] is able to reproduce accurately the differential capacitance of the $[\mathrm{BMI}]\left[\mathrm{PF}_{6}\right]$-graphite system. Moreover, the ionic densities predicted by this model (see figure 5) have the same characteristics as the ones obtained by all-atom simulations [17, 19]. Specifically, high density peaks are observed near the planar graphite electrodes and layers can be defined, centered at approximately $5 \AA, 10 \AA$ and $15 \AA$ from the surface at $\Delta \Psi^{0}=0.0 \mathrm{~V}$. When the potential difference increases, the layering is modified, and an alternation of anionic and cationic layers appears, which corresponds to an overscreening effect [10].

Kislenko et al. report on a two-dimensional structure present at the interface between the uncharged graphite electrodes and the ionic liquid [17]. This type of ordering in the first layer is confirmed experimentally for $[\mathrm{BMI}]\left[\mathrm{PF}_{6}\right]$ on mica [47] and was also demonstrated at the interface between $\mathrm{LiCl}$ and aluminium electrodes [34, 35]. A two-dimensional ordering in the first layer should be reflected by a sharp-peak in the in-plane partial anion-anion and cation-cation structure factors.

The $\mathrm{PF}_{6}^{-}-\mathrm{PF}_{6}^{-}$and $\mathrm{BMI}^{+}-\mathrm{BMI}^{+}$(for $\mathrm{BMI}^{+}$, the central site is considered, which corresponds to the imidazolium ring) partial structure factors calculated for the ions in the first adsorbed layer and corresponding snapshots are given in figure6. When there is no potential difference, it is clear from the partial structure factors that there is a two-dimensional ordering at the interface, not present in the bulk. The first layer is organized in the same defective hexagonal lattice as for the all-atom model [17], with a lattice spacing of approximately $10 \AA$. According to the snapshots and partial structure factors, the first layer becomes disordered 

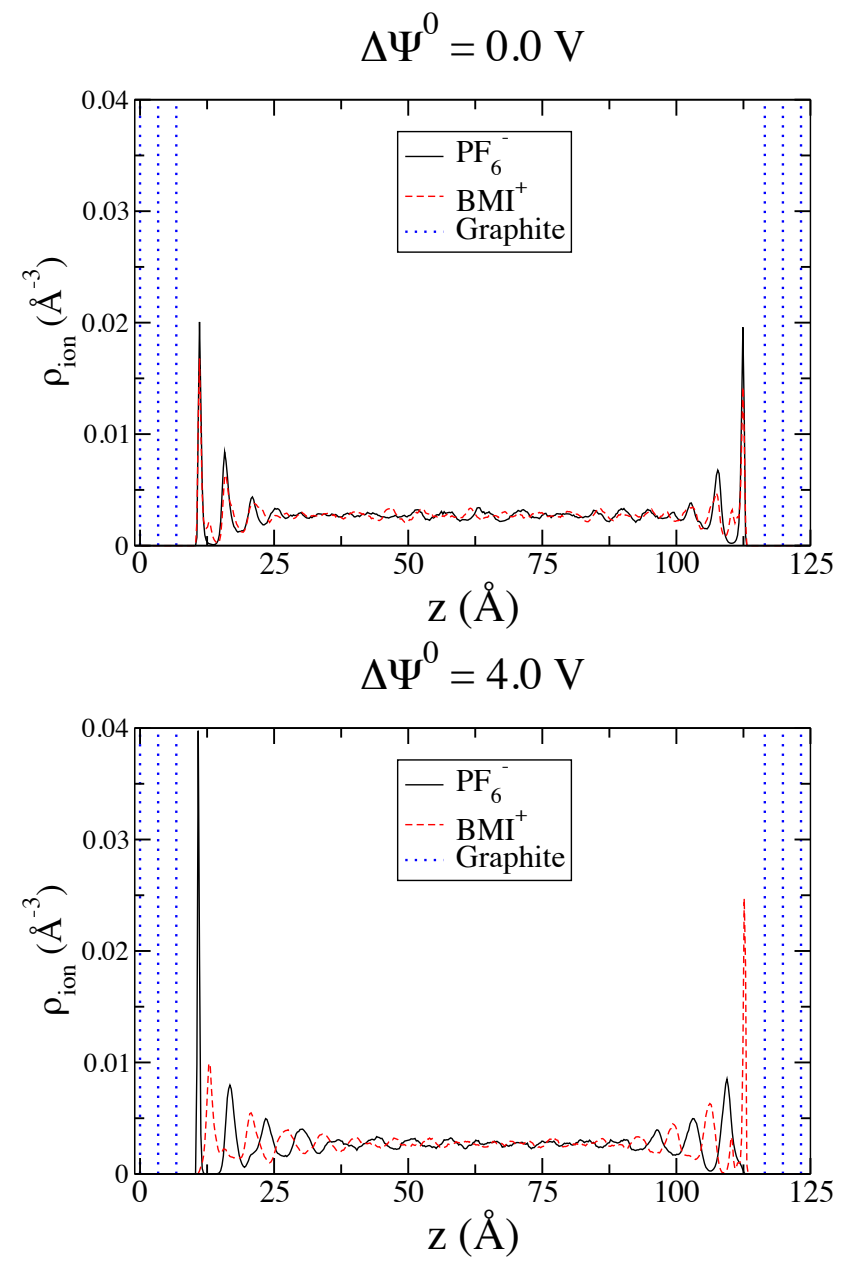

Figure 5: Ionic densities of anions and mass centers of cations for $\Delta \Psi^{0}=0.0 \mathrm{~V}$ and $\Delta \Psi^{0}=4.0 \mathrm{~V}$. When the potential difference is not equal to $0.0 \mathrm{~V}$, an asymmetry can be seen for the positive and negative electrodes.

when a potential difference is applied between the electrodes.

\section{CONCLUSION}

A coarse-grained model developed by Roy and Maroncelli 22 to represent the ionic liquid $[\mathrm{BMI}]\left[\mathrm{PF}_{6}\right]$ has been tested for the prediction of interfacial properties. It appears that the model is able to reproduce accurately the liquid-vapor interface as demonstrated by the good agreement between the calculated surface tension $\left(39.4 \mathrm{mN} . \mathrm{m}^{-1}\right.$ at $\left.400 \mathrm{~K}\right)$ and the experimental one $\left(40.8 \mathrm{mN} \cdot \mathrm{m}^{-1}\right.$ at $393 \mathrm{~K}$ [37], $41.07 \mathrm{mN} \cdot \mathrm{m}^{-1}$ at $343.15 \mathrm{~K}$ [38]). The model 

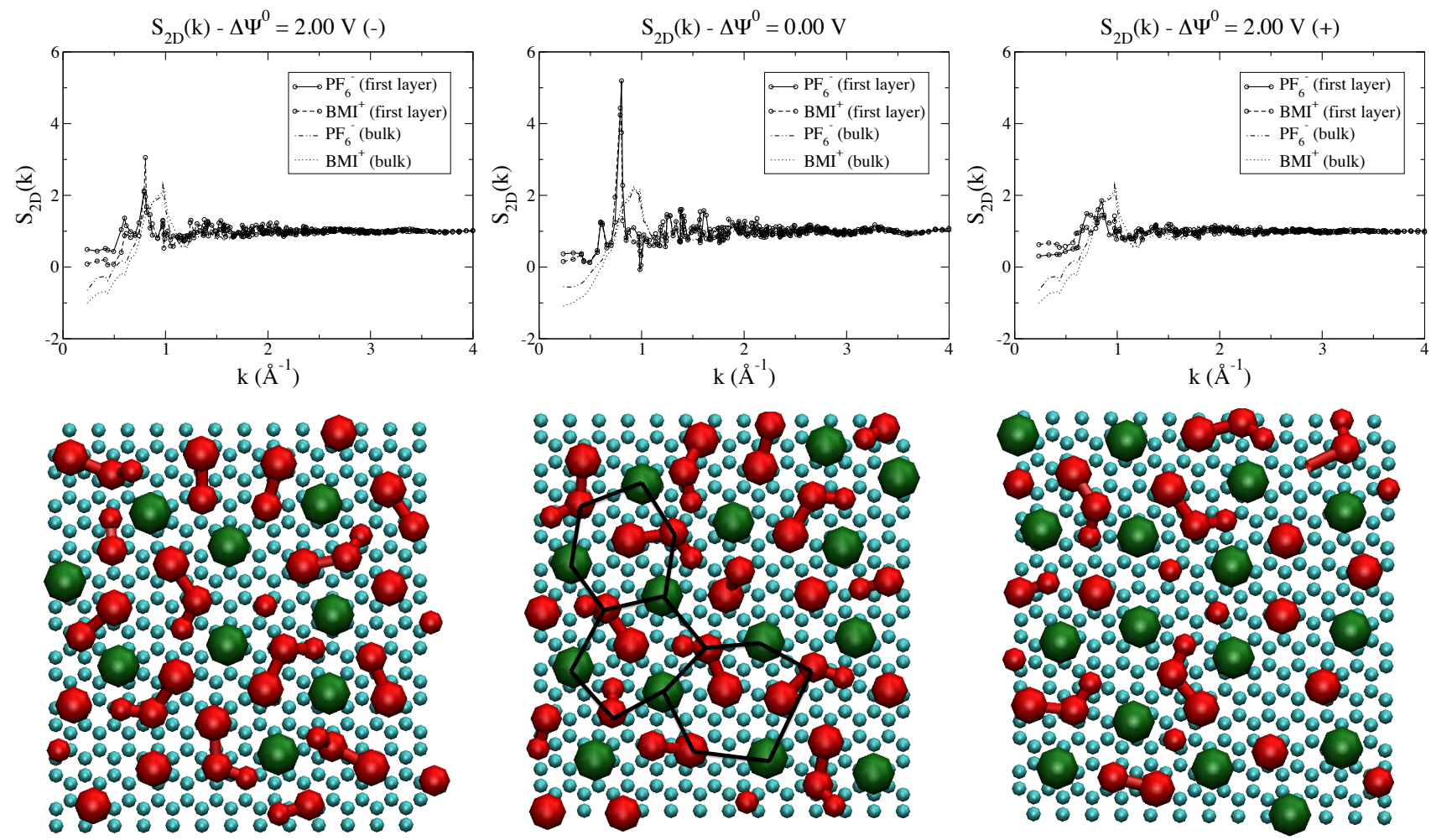

Figure 6: Upper panel: $\mathrm{PF}_{6}^{-}-\mathrm{PF}_{6}^{-}$and $\mathrm{BMI}^{+}-\mathrm{BMI}^{+}$partial structure factors for the ions in the first layer. For $\mathrm{BMI}^{+}$, the site considered in the calculation is the central site corresponding to the imidazolium ring. Lower panel: Corresponding representative snapshots, anions are in green, cations are in red and the graphite electrode is represented in blue. Black lines are added in the free potential snapshot to ease the visualization of the hexagonal lattice.

also gives qualitative and quantitative properties in agreement with all-atom simulations for liquid-graphite interfaces. In particular, the differential capacitances determined in this work (3.9 $\mu \mathrm{F} . \mathrm{cm}^{-2}$ for the positive electrode and $4.8 \mu \mathrm{F} . \mathrm{cm}^{-2}$ for the negative electrode) agree very well with all-atom simulations of Kislenko et al. $\left(3.7 \mu \mathrm{F} . \mathrm{cm}^{-2}\right.$ for the positive electrode and $4.6 \mu \mathrm{F} . \mathrm{cm}^{-2}$ for the negative electrode). In light of the satisfying results obtained in this work, the coarse-grained model for $[\mathrm{BMI}]\left[\mathrm{PF}_{6}\right]$ tested here appears as a suitable model for the study of the interface between $[\mathrm{BMI}]\left[\mathrm{PF}_{6}\right]$ and porous electrodes by simulations. Molecular dynamics simulations with polarizable nanoporous carbon electrodes will provide information to go beyond mean-field approaches [48] and recent simulations [49] which propose theories to explain the anomalous capacitance increase in these materials. 


\section{ACKNOWLEDGMENTS}

The authors acknowledge the support of the French Agence Nationale de la Recherche (ANR) under grant ANR-2010-BLAN-0933-02 ("Modeling the Ion Adsorption in Carbon Micropores").

[1] Lewandowski, A. and Galinski, M. (2004) J. Phys. Chem. Solids, 65(2-3), 281-286.

[2] Chmiola, J., Yushin, G., Gogotsi, Y., Portet, C., Simon, P., and Taberna, P. L. (2006) Science, 313(5794), 1760-1763.

[3] McEwen, A. B., McDevitt, S. F., and Koch, V. R. (1997) J. Electrochem. Soc., 144(4), L84L86.

[4] Chmiola, J., Largeot, C., Taberna, P.-L., Simon, P., and Gogotsi, Y. (2008) Angew. Chem. Int. Edit., 47(18), 3392-3395.

[5] Lewandowski, A., Olejniczak, A., Galinski, M., and Stepniak, I. (2010) J. Power Sources, 195(17), 5814-5819.

[6] Simon, P. and Gogotsi, Y. (2008) Nat. Mater., 7(11), 845-854.

[7] Simon, P. and Gogotsi, Y. (2010) Phil. Trans. R. Soc. A, 368(1923), 3457-3467.

[8] Alam, M. T., Islam, M. M., Okajima, T., and Ohsaka, T. (2007) J. Phys. Chem. C, 111(49), $18326-18333$.

[9] Alam, M. T., Islam, M. M., Okajima, T., and Ohsaka, T. (2008) J. Phys. Chem. C, 112(42), $16600-16608$.

[10] Kornyshev, A. A. (2007) J. Phys. Chem. B, 111(20), 5545-5557

[11] Fedorov, M., Georgi, N., and Kornyshev, A. (2010) Electrochem. Commun., 12(2), 296-299.

[12] Silva, F., Gomes, C., Figueiredo, M., Costa, R., Martins, A., and Pereira, C. M. (2008) J. Electroanal. Chem., 622(2), 153-160.

[13] Drüschler, M., Huber, B., and Roling, B. (2011) J. Phys. Chem. C, 115(14), 6802-6808.

[14] Bhargava, B. L. and Balasubramanian, S. (2007) J. Chem. Phys., 127(11), 114510.

[15] Dommert, F., Schmidt, J., Qiao, B., Zhao, Y., Krekeler, C., Site, L. D., Berger, R., and Holm, C. (2008) J. Chem. Phys., 129(22), 224501.

[16] Shah, J. K., Brennecke, J. F., and Maginn, E. J. (2002) Green Chem., 4, 112-118. 
[17] Kislenko, S. A., Samoylov, I. S., and Amirov, R. H. (2009) Phys. Chem. Chem. Phys., 11, $5584-5590$.

[18] Vatamanu, J., Borodin, O., and Smith, G. D. (2011) J. Phys. Chem. B, 115(12), 3073-3084.

[19] Feng, G., Qiao, R., Huang, J., Dai, S., Sumpter, B. G., and Meunier, V. (2011) Phys. Chem. Chem. Phys., 13, 1152-1161.

[20] Fedorov, M. V. and Kornyshev, A. A. (2008) J. Phys. Chem. B, 112(38), 11868-11872.

[21] Pastewka, L., Järvi, T. T., Mayrhofer, L., and Moseler, M. (2011) Phys. Rev. B, 83(16), 165418.

[22] Roy, D. and Maroncelli, M. (2010) J. Phys. Chem. B, 114(39), 12629-12631.

[23] Reed, S. K., Lanning, O. J., and Madden, P. A. (2007) J. Chem. Phys., 126(8), 084704.

[24] Roy, D., Patel, N., Conte, S., and Maroncelli, M. (2010) J. Phys. Chem. B, 114(25), 8410-8424

[25] Lynden-Bell, R. M. and Youngs, T. G. A. (2009) J. Phys.: Condens. Matter, 21(42), 424120.

[26] Chaumont, A., Schurhammer, R., and Wipff, G. (2005) J. Phys. Chem. B, 109(40), 1896418973

[27] Chevrot, G., Schurhammer, R., and Wipff, G. (2006) Phys. Chem. Chem. Phys., 8, 4166-4174.

[28] Leontyev, I. and Stuchebrukhov, A. (2011) Phys. Chem. Chem. Phys., 13, 2613-2626.

[29] Chaban, V. V., Voroshylova, I. V., and Kalugin, O. N. (2011) Phys. Chem. Chem. Phys., 13, $7910-7920$

[30] Cole, M. W. and Klein, J. R. (1983) Surf. Sci., 124(2-3), 547-554.

[31] Siepmann, J. I. and Sprik, M. (1995) J. Chem. Phys., 102(1), 511-524.

[32] Kolafa, J. (2004) J. Comput. Chem., 25(3), 335-342.

[33] Kühne, T. D., Krack, M., Mohamed, F. R., and Parrinello, M. (2007) Phys. Rev. Lett., 98(6), 066401.

[34] Pounds, M., Tazi, S., Salanne, M., and Madden, P. A. (2009) J. Phys.: Condens. Matter, 21(42), 424109.

[35] Tazi, S., Salanne, M., Simon, C., Turq, P., Pounds, M., and Madden, P. A. (2010) J. Phys. Chem. B, 114(25), 8453-8459

[36] Gingrich, T. R. and Wilson, M. (2010) Chem. Phys. Lett., 500(1-3), 178-183.

[37] Ghatee, M. H. and Zolghadr, A. R. (2008) Fluid Phase Equilibr., 263(2), 168-175.

[38] Freire, M. G., Carvalho, P. J. ., Fernandes, A. M., Marrucho, I. M., Queimada, A. J., and Countinho, J. A. P. (2007) J. Colloid Interf. Sci., 314(2), 621-630. 
[39] Heggen, B., Zhao, W., Leroy, F., Dammers, A. J., and Müller-Plathe, F. (2010) J. Phys. Chem. B, 114(20), 6954-6961.

[40] Salanne, M., Simon, C., Turq, P., and Madden, P. A. (2007) C. R. Chimie, 10(10-11), 11311136.

[41] Frenkel, D. and Smit, B. (2001) Understanding Molecular Simulation, Academic Press, Inc., Orlando, FL, USA 2nd edition.

[42] Gelfand, M. P. and Fisher, M. E. (1990) Physica A, 166(1), 1-74.

[43] Aguado, A., Scott, W., and Madden, P. A. (2001) J. Chem. Phys., 115(18), 8612-8619.

[44] Gonzalez-Melchor, M., Bresme, F., and Alejandre, J. (2005) J. Chem. Phys., 122(10), 104710.

[45] Canongia Lopes, J. N., Deschamps, J., and Pádua, A. A. H. (2004) J. Phys. Chem. B, 108(6), 2038-2047.

[46] Wang, S., Li, S., Cao, Z., and Yan, T. (2010) J. Phys. Chem. C, 114(2), 990-995.

[47] Liu, Y., Zhang, Y., Wu, G., and Hu, J. (2006) J. Am. Chem. Soc., 128(23), 7456-7457.

[48] Kondrat, S. and Kornyshev, A. (2011) J. Phys.: Condens. Matter, 23(2), 022201.

[49] Shim, Y. and Kim, H. J. (2010) ACS Nano, 4(4), 2345-2355 
Graphical asbtract

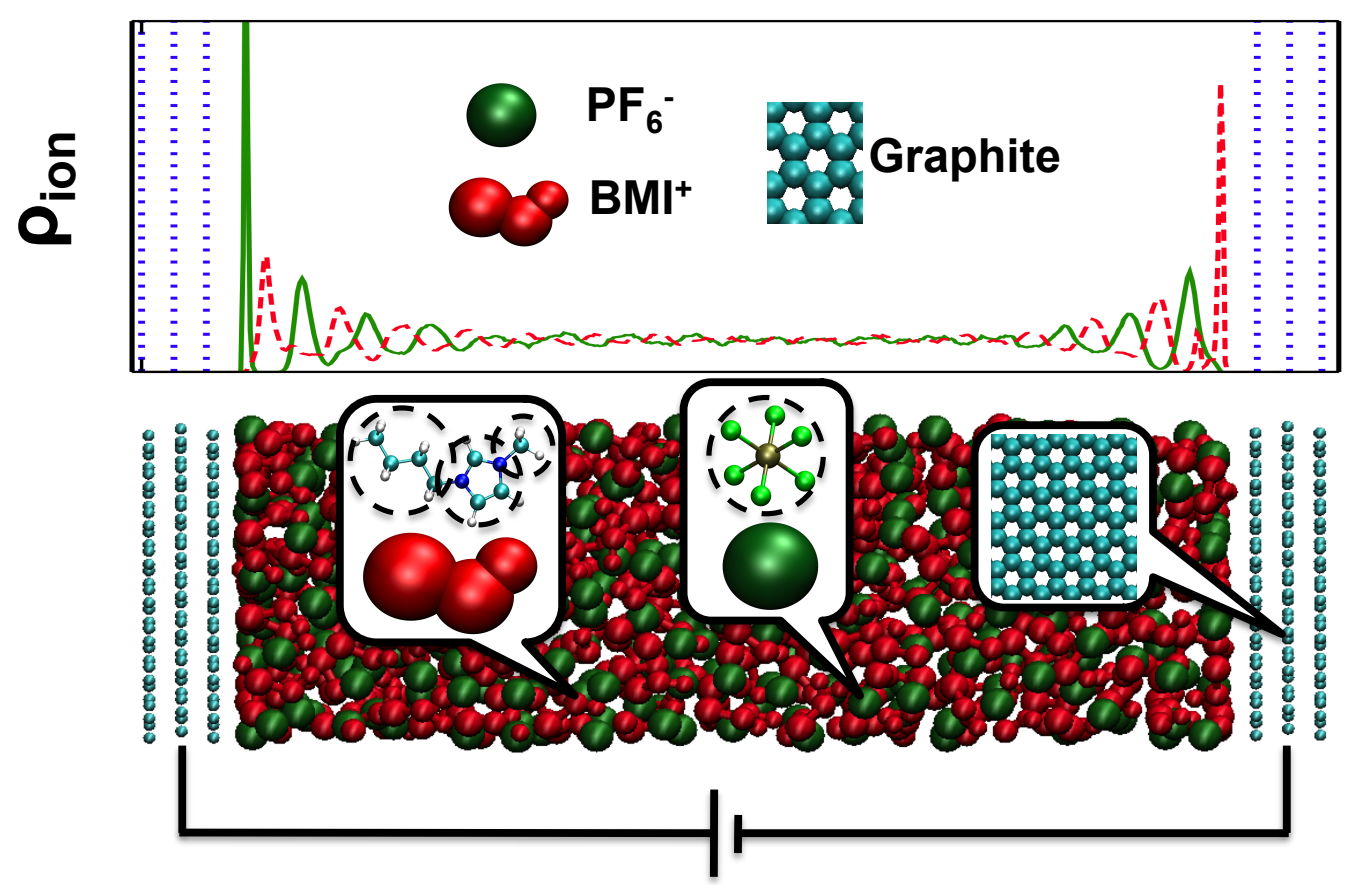

